\title{
PAPER \\ An Adaptive Fairness and Throughput Control Approach for Resource Scheduling in Multiuser Wireless Networks
}

\author{
Lin SHAN $^{\dagger \text { a) }}$, Member, Sonia AÏSSA ${ }^{\dagger \dagger}$, Nonmember, Hidekazu MURATA ${ }^{\dagger}$, Senior Member, \\ Susumu YOSHIDA ${ }^{\dagger}$, Fellow, and Liang $\mathrm{ZHAO}^{\dagger}$, Member
}

\begin{abstract}
SUMMARY The important issue of an adaptive scheduling scheme is to maximize throughput while providing fair services to all users, especially under strict quality of service requirements. To achieve this goal, we consider the problem of multiuser scheduling under a given fairness constraint. A novel Adaptive Fairness and Throughput Control (AFTC) approach is proposed to maximize the network throughput while attaining a given min-max fairness index. Simulation results reveal that comparing to straightforward methods, the proposed AFTC approach can achieve the desired fairness while maximizing the throughput with short convergence time, and is stable in dynamic scenarios. The trade-off between fairness and throughput can be accurately controlled by adjusting the scheduler's parameters.

key words: multiuser diversity, multiuser scheduling, proportional fairness, fairness index, adaptive fairness, throughput control
\end{abstract}

\section{Introduction}

Multiuser diversity is a fundamental characteristic of multiuser network established by the independently timevarying fading channels among users. This diversity can be exploited to improve the throughput performance by opportunistically scheduling users on favorable resources [1]. However, focusing only on the possible diversity gain can lead to unfair treatment of individual users. If the system has a certain fairness constraint such as in emergency situations, e.g., in a major natural disaster, providing a target fairness such as equal throughput allocation to users is more important. For an adaptive and efficient scheduling scheme, we need a mechanism of adjustable fairness and throughput [2], [3].

For the purpose of controlling the trade-off between network throughput and fairness, various scheduling schemes have been proposed and used in many applications. The proportional fairness (PF) scheduling scheme is a notable algorithm that can maximize the logarithmic total throughput by exploiting multiuser diversity [4]. A modified PF scheme called weighted PF (WPF) can control the throughput-fairness trade-off by adjusting the scheduler's parameter [5]. Recently, a scheduling and resource sharing scheme referred to as adaptive PF (APF) was proposed in [6], [7], that maps the channel quality of each user into a

Manuscript received February 22, 2012.

Manuscript revised August 21, 2012.

${ }^{\dagger}$ The authors are with the Graduate School of Informatics, Kyoto University, Kyoto-shi, 606-8501 Japan.

${ }^{\dagger}$ The author is with the INRS-EMT, University of Québec, Montreal, QC, Canada.

a) E-mail: contact-h24@ hanase.kuee.kyoto-u.ac.jp

DOI: 10.1587/transcom.E96.B.561 defined utility function and controls the throughput-fairness trade-off in a more adaptive and efficient way.

These existing scheduling schemes can provide some control on the trade-off between throughput and fairness. However, it is not trivial to apply them to obtain a given fairness target, because they lack the features to precisely control the system fairness performance. Moreover, they cannot provide a robust control of fairness in dynamic scenarios [8]. For example, varying the number of users or their locations may lead to significantly different results. In order to achieve a given fairness, a natural solution for WPF and APF is to adjust the scheduler's parameter using binary search. However, such a straightforward method is not efficient, because the convergence time is long and it is ineffective in dynamic scenarios.

Therefore, Schwarz et al. proposed a downlink multiuser scheduling scheme based on WPF to obtain the desired fairness specified in terms of the Jain fairness index [3]. However, their approach is designed only for the Jain fairness index. On the other hand, we consider that the minmax fairness index [9] is more appropriate, especially in emergency situations, because the Jain fairness index [10] and other indexes such as the Gini index [11] might ignore the minor lowest-throughput users. Moreover, comparing to WPF, APF is a newer and better scheme. Therefore, this work aims at an APF-based scheduling scheme of adjustable fairness and throughput.

In this paper, we propose a novel adaptive fairness and throughput control (AFTC) approach based APF, to maximize the network throughput while attaining a given fairness quantified by the min-max fairness index. In the proposed AFTC approach, an updating module is introduced to track the data rate allocated to users and update the scheduler's parameters. The purpose of this approach is to make the achieved fairness index equal to the desired index and assure robustness in dynamic scenarios. The trade-off between fairness and throughput can be accurately controlled by the given fairness index.

The remainder of this paper is organized as follows. Section 2 describes the proportional fairness based multiuser scheduling schemes and fairness measurement. Section 3 describes the proposed AFTC approach. Section 4 presents the system assumption and the simulation results. Section 5 includes our concluding remarks. 


\section{Proportional Fairness Based Scheduling and Fair- ness Measurement}

In this section, we describe the above-mentioned proportional fairness (PF) based multiuser scheduling schemes and present a general definition of fairness in the resource allocation to the users. Herein, we assume that the scheduler selects only one user in each transmission time interval (TTI), which is equal to the length of one transmission frame. The complex channel gains for the fading links from all users $h_{n}, n \in\{1, \ldots, N\}$ to the serving base station (BS) are assumed to be i.i.d. $C \mathcal{N}(0,1)$, i.e., distributed according to Rayleigh model, and to be constant within each TTI, where $N$ is the total number of users.

\subsection{Proportional Fairness Based Scheduling}

The user selection criterion according to the Weighted Proportional Fairness (WPF) scheduling scheme is given by [5]:

$$
n^{*}=\underset{n \in\{1, \ldots, N\}}{\arg \max } \frac{\left(r_{n}\right)^{e}}{R_{n}},
$$

where $r_{n}$ is the instantaneous rate of user $n, R_{n}$ is the average achievable rate of user $n$, and $e$ is the exponent parameter used to control the trade-off between throughput and fairness. A special case of the WPF scheduling scheme when $e=1$ in (1), corresponds to the original PF scheduling scheme [4]. The instantaneous rate of user $n$ can be expressed as

$$
r_{n}=\log _{2}\left(1+\left|h_{n}\right|^{2} \gamma_{n}\right),
$$

where $\gamma_{n}$ denotes the average received signal-to-noise ratio (SNR) at the BS for the link between user $n$ and the BS.

The average achievable rate $R_{n}$ is updated at each TTI according to the following rule:

$$
\begin{cases}R_{n}=\left(1-\frac{1}{L}\right) R_{n}+\frac{1}{L} r_{n}, & \text { if } n=n^{*} \\ R_{n}=\left(1-\frac{1}{L}\right) R_{n}, & \text { if } n \neq n^{*},\end{cases}
$$

where $L(0<1 / L<1)$ is the average time window size over which fairness is reflected.

Since the exponent parameter $e$ in (1) takes a fixed value for all users, this approach gives rise to two problems, demonstrated in [6]: (i) The time-varying diversity of each user cannot be adapted because of the exponent parameter being fixed in time, and (ii) It is not possible to fix a certain value for $e$ that ensures fairness among all users at the same time because this parameter takes a unique value for all users.

Tackling these issues, a modified PF based multiuser scheduling called adaptive proportional fairness (APF) was proposed in [6], in which the user selection criterion is given by:

$$
n^{*}=\underset{n \in\{1, \ldots, N\}}{\arg \max } \frac{\left(r_{n}\right)^{c_{n}}}{R_{n}},
$$

where $c_{n}$ is an independent exponent parameter corresponding to user $n$. This parameter is particular to each user and determined in order to avoid the dependency between different users. The updating of the average achievable rate $R_{n}$ is as shown in (3).

To achieve long-term fairness among users, the updating of each user's exponent parameter $c_{n}$ is done at a larger time-scale. Indeed, the updating of the exponent parameters is done only when necessary for achieving proportional fairness among users. The updating module verifies whether the difference between normalized throughput, defined as $R_{n} / \bar{r}_{n}$, and the average value of all users is within acceptable values defined by the interval $[-\varepsilon, \varepsilon]$. Parameter $\bar{r}_{n}$ represents the average achievable channel capacity of user $n$, and $\varepsilon$ is a fairness tuning parameter that defines the degree of fairness requirement. Herein, the exponent parameter $c_{n}$ is updated according to [6]:

$$
\begin{cases}c_{n}=c_{n}+\Delta c, & \text { if }\left(\frac{R_{n}}{\bar{r}_{n}}-\frac{1}{N} \sum_{n=1}^{N} \frac{R_{n}}{\bar{r}_{n}}\right)<-\varepsilon \\ c_{n}=c_{n}-\Delta c, & \text { if }\left(\frac{R_{n}}{\bar{r}_{n}}-\frac{1}{N} \sum_{n=1}^{N} \frac{R_{n}}{\bar{r}_{n}}\right)>\varepsilon,\end{cases}
$$

where the choice of $\Delta c$ depends on the speed of the convergence requirement. We note that when the value of $\varepsilon$ is large enough such as $\varepsilon=100$, no updating of each user's exponent parameter $c_{n}$ is performed, and the fairness and throughput achieved by APF completely depends on the initial values of parameters $c_{n}, n=1, \ldots, N$.

\subsection{Fairness Measurement}

In multiuser networks, the measurement of fairness has been extensively studied in the literature. In this paper, we rely on a general fairness measure called min-max fairness index, which has been usually used to measure the achieved fairness of resource scheduling schemes. The min-max fairness index is defined as the ratio of the minimum and the maximum throughput allocated to users, and is given by [9]:

$$
f_{\text {min-max }}=\frac{\min \left\{\hat{T}_{n}\right\}}{\max \left\{\hat{T}_{n}\right\}}, \quad n \in\{1, \ldots, N\},
$$

where $\hat{T}_{n}$ is the achieved total throughput of user $n$. The numerator of (6) expresses the smallest throughput allocated among users, and the denominator expresses the largest throughput allocated among users. The value of the minmax fairness index $f_{\min -\max }$ is between 0 and 1 . Note that $f_{\text {min-max }}=0$ expresses extremely unfair resource allocation to the users, whereas $f_{\min -\max }=1$ indicates a perfectly fair resource allocation to the users.

Note that this paper does not focus on the discussion in the definition of fairness index. We focus on that, given a certain index, how to guarantee it while achieving fast convergence and high throughput. This is a feature that existing 
scheduling schemes WPF and APF lack.

\section{Proposed Adaptive Fairness and Throughput Con- trol Scheme}

In this section, we consider the problem of multiuser scheduling under a given fairness constraint, and propose a novel adaptive fairness and throughput control (AFTC) approach to maximize the network throughput while attaining a given fairness quantified by the min-max fairness index. The proposed AFTC approach creates a relation between the min-max fairness index and the multiuser scheduling scheme.

We describe the proposed AFTC approach, which is based on the above-described APF scheduling scheme. The concept of the AFTC approach is illustrated in Fig. 1. In particular, the user selection criterion is given by:

$$
n^{*}=\underset{n \in\{1, \ldots, N\}}{\arg \max } \frac{\left(r_{n}\right)^{\psi_{n}}}{R_{n}}
$$

where $r_{n}, R_{n}$, and $\psi_{n}$ are the instantaneous rate of user $n$, the average achievable rate of user $n$, and the exponent parameter corresponding to user $n$, respectively. The update rule of the user's average achievable rate $R_{n}$ is the same as that of APF. However, the updating rule of $\psi_{n}$ is different from that of APF, which is the most important part for the fairness and throughput control that we target. The aim of the proposed approach is to make the achieved min-max fairness index

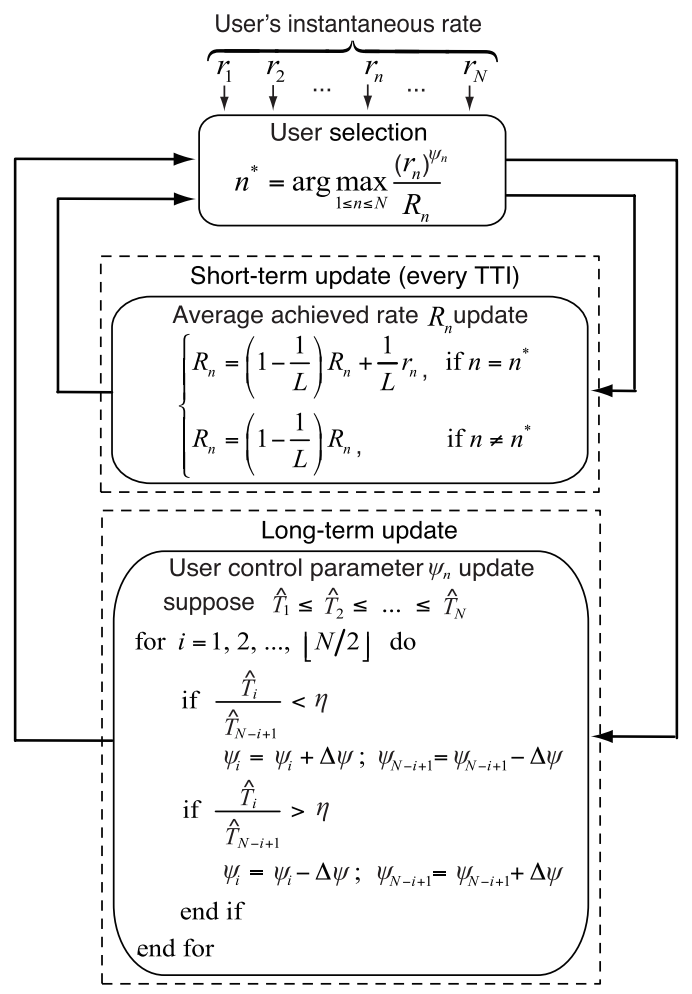

Fig. 1 The procedure of the Adaptive Throughput and Fairness Control (AFTC) approach, where $\eta$ is the desired min-max fairness index. $f_{\min -\max }\left(0 \leq f_{\min -\max } \leq 1\right)$ equal to the desired fairness index and maintain the total throughput at a high level. Here, we define $\eta(0 \leq \eta \leq 1)$ as the desired fairness specified in terms of the min-max fairness index.

In order to make the achieved fairness index $f_{\min -\max }$ equal to a desired fairness index $\eta$, the selection criterion in (7) should also be based on $\eta$. Since $r_{n}$ and $R_{n}$ do not have an explicit relation with $\eta$, we focus on the updating rule of each user's control parameter $\psi_{n}$. Herein, the updating rule of $\psi_{n}$ is designed based on the desired fairness index $\eta$. For instance, if the achieved fairness $f_{\min -\max }$ is less than the desired fairness $\eta$, that means some users are not served with enough resources while other get most of the resources. Therefore, we should increase the probability for selecting the former kind of users, and decrease the probability for selecting the users of the latter type. The selection probability of each user $n$, can be controlled by adjusting the value of $\psi_{n}$ shown in (7). Indeed, increasing the value of $\psi_{n}$ can increase the selection probability of user $n$ and decreasing the value of $\psi_{n}$ can decrease the selection probability of that user.

Now, we elaborate on the updating rule of $\psi_{n}$. To achieve long-term fairness among users, we consider that the value of $\psi_{n}$ is updated at a larger time-scale than that of $R_{n}$. Indeed, the updating of each user's $\psi_{n}$ is only performed if the achieved fairness $f_{\text {min-max }}$ has reached a stationary state. Such a stationary state is decided through measurement of the changes exhibited by the achieved fairness $f_{\text {min-max }}$ between consecutive TTIs. If $\mid f_{\min -\max }(t)-$ $f_{\min -\max }(t-1) \mid<\delta$, where $\delta$ is a system parameter which is decided by the system operator, then this indicates that $f_{\min -\max }$ is specified to have reached a stationary state. A small value of $\delta$ results in a long-term but precise decision, whereas a large value of $\delta$ results in a short-term but approximate decision. When the stationary state of $f_{\min \text {-max }}$ is reached, the user control parameter $\psi_{n}$ is updated using the following algorithm, where without loss of generality, we suppose $\hat{T}_{1} \leq \hat{T}_{2} \leq \ldots \leq \hat{T}_{N}$.

$$
\begin{aligned}
& \hline \begin{array}{l}
\text { User Control Parameter } \psi_{n} \text { Update Algorithm } \\
\hline \text { for } i=1,2, \ldots,\lfloor N / 2\rfloor \text { do }
\end{array} \\
& \text { if } \quad \frac{\hat{T}_{i}}{\hat{T}_{N-i+1}}<\eta \\
& \quad \psi_{i}=\psi_{i}+\Delta \psi ; \quad \psi_{N-i+1}=\psi_{N-i+1}-\Delta \psi \\
& \text { elseif } \frac{\hat{T}_{i}}{\hat{T}_{N-i+1}}>\eta \\
& \quad \psi_{i}=\psi_{i}-\Delta \psi ; \quad \psi_{N-i+1}=\psi_{N-i+1}+\Delta \psi \\
& \text { end if } \\
& \text { end for }
\end{aligned}
$$

The choice of $\Delta \psi$ depends on the required convergence speed. By increasing the value of $\Delta \psi$, faster convergence to the target fairness $\eta$ can be achieved but the convergence has more oscillations around this value. To avoid these oscillations and make a precise decision on achieved fairness, in 
our computer simulations, we assume $\Delta \psi=0.1$ and $\delta=0.1$.

Here we explain how to update the user control parameter $\psi_{n}$ to achieve the required fairness target and comparatively higher network throughput. We first discuss how to make the achieved min-max fairness equal to the target fairness $\eta$ using this update algorithm. If $\hat{T}_{i} / \hat{T}_{N-i+1}<\eta, \psi_{i}$ is updated to $\psi_{i}+\Delta \psi$ and $\psi_{N-i+1}$ is updated to $\psi_{N-i+1}-\Delta \psi$. The selection probability of user $i$ increases with increasing $\psi_{i}$ and decreases with decreasing $\psi_{i}$. Hence, $\hat{T}_{i}$ increases and $\hat{T}_{N-i+1}$ decreases after the updating of $\psi_{i}$ and $\psi_{N-i+1}$. Consequently, $\hat{T}_{i} / \hat{T}_{N-i+1}$ becomes large. In case $\hat{T}_{i} / \hat{T}_{N-i+1}>\eta, \psi_{i}$ is updated to $\psi_{i}-\Delta \psi$ and $\psi_{N-i+1}$ is updated to $\psi_{N-i+1}+\Delta \psi$. Hence, $\hat{T}_{i} / \hat{T}_{N-i+1}$ becomes small. As a result, the achieved min-max fairness $f_{\min -\max }$ can be equal to the target fairness $\eta$. In contrast to APF, the achieved fairness and throughput by AFTC do not depend on the initial value of each user's exponent parameter $\psi_{n}$, which is a major difference with the APF technique.

A straightforward method for updating $\psi$, would refer to the case when $i=1$ only. However, it does not exploit the temporal and multiuser diversities and this may lead to poor throughput performance. For example, if the instantaneous rate of user with throughput $\hat{T}_{1}$ is very low, the network overall throughput is also low. By considering all user pairs that can exploit full temporal and multiuser diversities, because parameter $\psi_{n}$ has a strong relation with user selection part (7) and updating $\psi_{n}$ can control the user's selection priority. Thus, we can achieve not only desired fairness, but also improved network throughput.

\section{Simulation Results}

In this section, we show simulation results using the proposed AFTC technique and compare the results with those of several conventional schemes both in dynamic and static scenarios.

We assume a full buffer traffic model in which each user (or base station) has packets awaiting transmission to base station (or users). The maximum possible system throughput can be evaluated for the full buffer traffic model, reflecting the multiuser and temporal diversity gain on the throughput performance. We consider a block Rayleigh fading channel model with independent channel realizations and take into account the path loss with exponent $\alpha=3$. For the sake of simplicity, the effect of shadowing is not considered. The users are assumed to be randomly and uniformly located in a cell area, and the total number of users $N$ in a cell is variable in different simulation situations. The cell radius is normalized to 1 , and the cell edge average SNR is assumed to be $0 \mathrm{~dB}$. We assume that the initial value of each user's exponent parameter $\psi_{n}$ in AFTC and $c_{n}$ in APF, are equal to 1 unless otherwise mentioned, which corresponds to the original PF method.

\subsection{Performance Evaluation in Dynamic Scenario}

We first investigate the effects of the users' location and
Table 1 Comparison of achieved min-max fairness index between the proposed AFTC approach and two PF based scheduling schemes in different users' location cases, where the number of users is fixed to $N=10$.

\begin{tabular}{|l|c|c|c|c|c|c|}
\hline \multirow{2}{*}{$\begin{array}{l}\text { Users' } \\
\text { location }\end{array}$} & \multicolumn{2}{|c|}{ AFTC } & \multicolumn{2}{c|}{ WPF } & \multicolumn{2}{c|}{ APF } \\
\cline { 2 - 7 } & $\eta=0.5$ & $\eta=0.9$ & $e=1$ & $e=0.1$ & $\varepsilon=1$ & $\varepsilon=0.1$ \\
\hline Case 1 & 0.50 & 0.90 & 0.41 & 0.82 & 0.77 & 0.91 \\
Case 2 & 0.49 & 0.89 & 0.25 & 0.78 & 0.80 & 0.90 \\
Case 3 & 0.51 & 0.91 & 0.38 & 0.81 & 0.72 & 0.94 \\
Case 4 & 0.51 & 0.91 & 0.53 & 0.77 & 0.68 & 0.92 \\
Case 5 & 0.49 & 0.90 & 0.31 & 0.92 & 0.66 & 0.87 \\
Case 6 & 0.50 & 0.90 & 0.43 & 0.69 & 0.71 & 0.91 \\
Case 7 & 0.50 & 0.90 & 0.36 & 0.92 & 0.78 & 0.93 \\
Case 8 & 0.50 & 0.90 & 0.42 & 0.76 & 0.81 & 0.92 \\
Case 9 & 0.49 & 0.89 & 0.16 & 0.83 & 0.70 & 0.85 \\
Case 10 & 0.50 & 0.90 & 0.26 & 0.76 & 0.69 & 0.88 \\
\hline
\end{tabular}

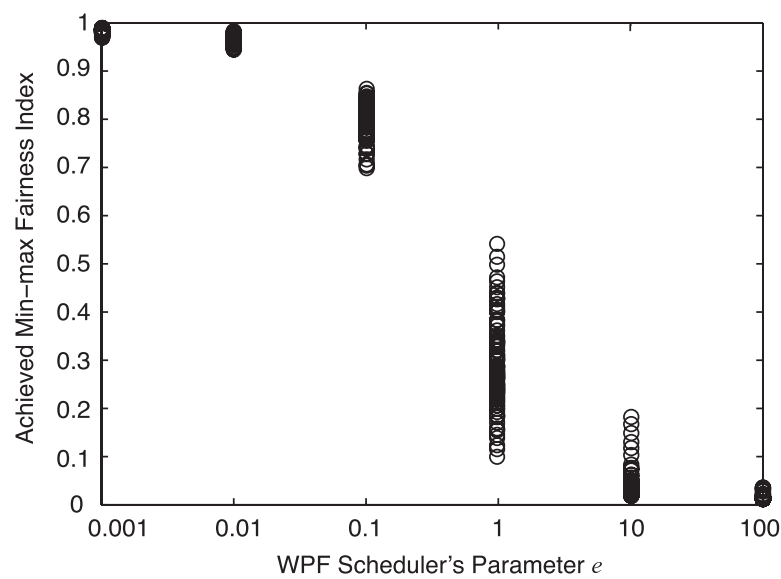

Fig. 2 Fairness performance of WPF where the number of users is fixed to $N=10$. For each control parameter $e \geq 0$, the results are calculated for 100 different users' locations.

the scheduler's parameters on the achieved fairness performance of the proposed AFTC, the WPF and the APF scheduling schemes. Table 1 shows a comparison of the achieved fairness index by each scheduling scheme for different cases of users' location, where the number of users is fixed to $N=10$. $\eta, e$ and $\varepsilon$ are the scheduler parameters of AFTC, WPF and APF, respectively, and the achieved fairness index can be controlled by adjusting these values. The achieved fairness is quantified by the min-max fairness index and calculated by taking 10,000 frames until the achieved fairness index becomes stable for each location case. From Table 1, we find that the achieved fairness index of AFTC is almost equal to the value of $\eta$ regardless of changing the users' location. However, for WPF and APF, although the values of the WPF parameter $e$ and the APF parameter $\varepsilon$ are fixed, the achieved fairness index changes when varying the users' locations.

To analyze the achieved fairness performance of AFTC, WPF and APF in more details, Figs. 2, 3 and 4 show the achieved fairness performance of each scheduling scheme with different values of the scheduler's parameter and the users' locations, where we measure the fairness for users' 100 different locations.

For the WPF scheduling scheme shown in Fig. 2, we 


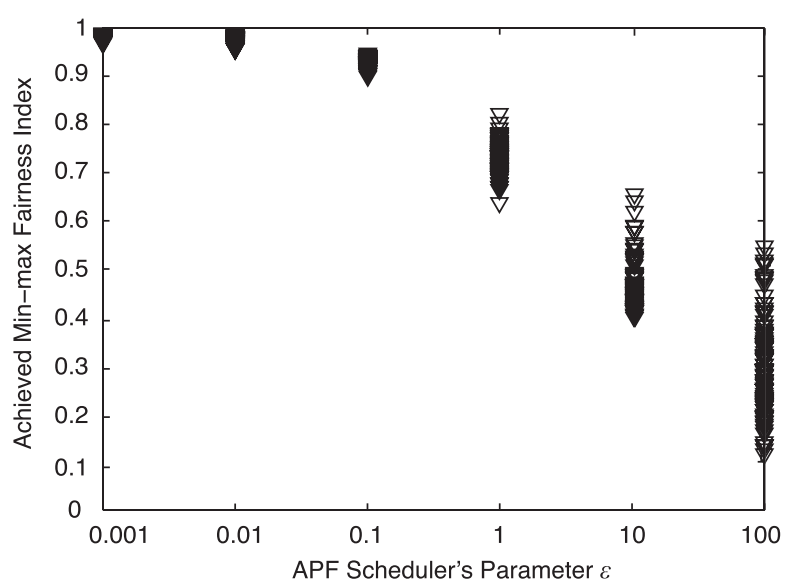

Fig. 3 Fairness performance of APF where the number of users is fixed to $N=10$. For each control parameter $\varepsilon \geq 0$, the results are calculated for 100 different users' locations.

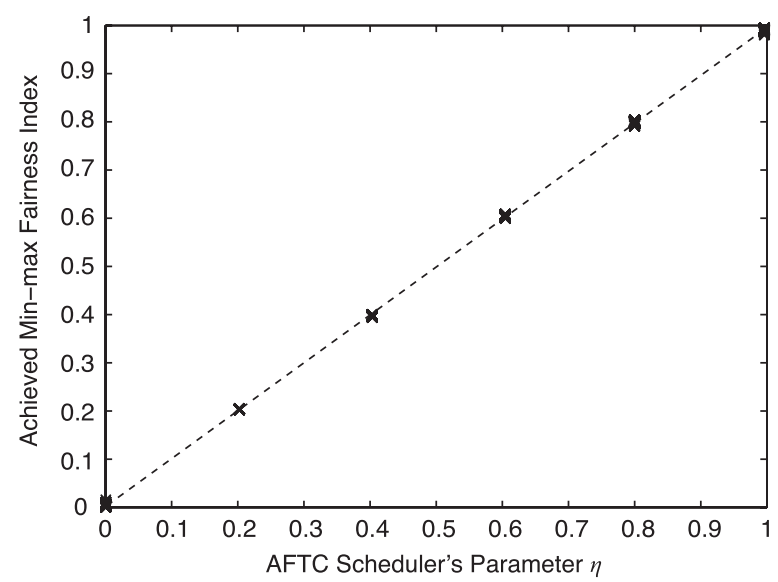

Fig. 4 Achieved fairness performance vs. desired fairness $\eta$ by applying the proposed AFTC approach. The number of users is $N=10$ and the results are calculated for 100 different users' locations.

observe that the achieved min-max fairness index decreases with increasing value of $e$. The achieved fairness index can be approximately controlled by adjusting the value of $e$. However, we find that even when the value of the WPF parameter $e$ is fixed, the achieved fairness index changes by changing the users' locations in the cases for $0.001 \ll$ $e \ll 100$. The achieved fairness becomes stable only when $e=0.001$ and $e=100$. As a result, it is very difficult to apply WPF to obtain a desired fairness in dynamic scenarios such as varying users' locations.

For the APF scheduling scheme considered in Fig. 3, the achieved min-max fairness index decreases when increasing value of the APF parameter $\varepsilon$. We observe that the dispersion of the achieved fairness index increases with increasing $\varepsilon$. This result means that the fairness control becomes non-robust with larger $\varepsilon$. Only for the case when $\varepsilon=0.001$, the achieved fairness becomes stable and close to 1 , which expresses perfect fairness performance. Since APF also cannot provide a desired fairness and a robust fairness control in such a dynamic scenario, it is unreasonable

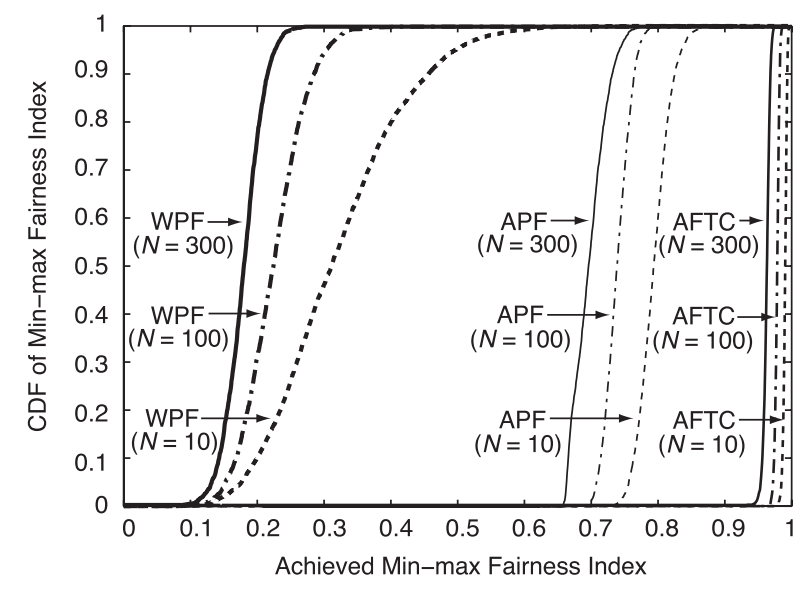

Fig. 5 CDF of achieved min-max fairness index applying the proposed AFTC with $\eta=1$, WPF with $e=1$, and APF with $\varepsilon=1$. The results are calculated for variable number of users and 1000 different users' locations.

to apply the WPF and the APF scheduling schemes to obtain a given fairness, because these schemes lack the features to achieve that.

Figure 4 shows the achieved fairness performance of our proposed AFTC approach, where we measure the fairness quantified by the min-max fairness index for users' 100 different locations and $N=10$. We observe that the minmax fairness index achieved by applying AFTC is almost equal to any desired fairness index $\eta$ for different scenarios of users' locations. This result demonstrates that our proposed AFTC approach can provide any desired min-max fairness index and a robust fairness control in dynamic scenarios.

Next, we consider that the number and location of users are both variable at a cell area in the simulation assumption. We use the cumulative distribution function (CDF) to calculate the probability of the achieved fairness index. Figure 5 shows the CDF of the achieved min-max fairness index when applying the following schemes: the proposed AFTC with $\eta=1$, WPF with $e=1$, and APF with $\varepsilon=1$. We calculate the results for a variable number of users $N$ and 1000 different users' locations. We observe that for the WPF, the value of the achieved fairness index decreases as the number of users $N$ increases, and the dispersion of achieved fairness indexes increases with decreasing the number of users. As a result, it is very difficult to provide a stable fairness service using WPF, especially when the number of users in a cell is small. For APF, the value of the achieved fairness index also decreases with increasing number of users, but the dispersion of the achieved fairness indexes does not significantly change when varying the number of users. The proposed AFTC can provide a stable fairness index regardless of the impact of varying the number and the location of users.

As a conclusion, in order to guarantee a desired fairness index, WPF and APF require nontrivial additional mechanism to adapt to the dynamic scenarios, whereas AFTC can automatically do the adaptation. We note that, due to this 


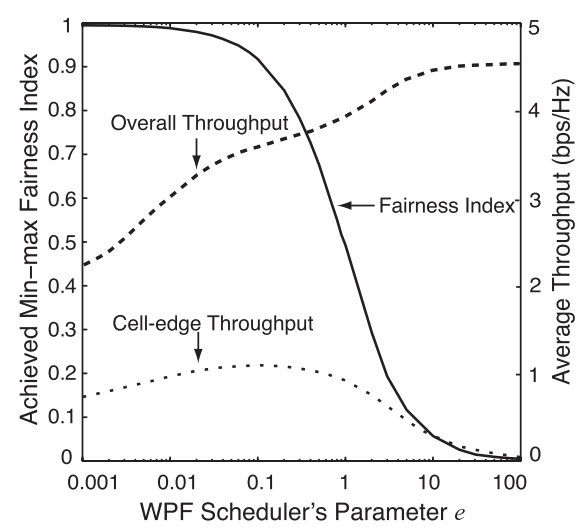

(a) WPF scheduling scheme

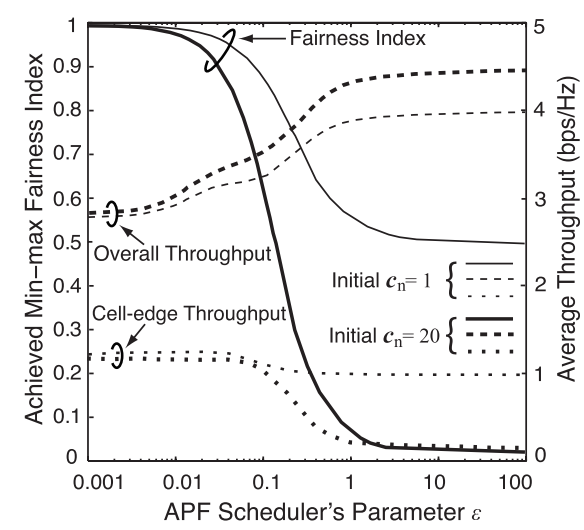

(b) APF scheduling scheme

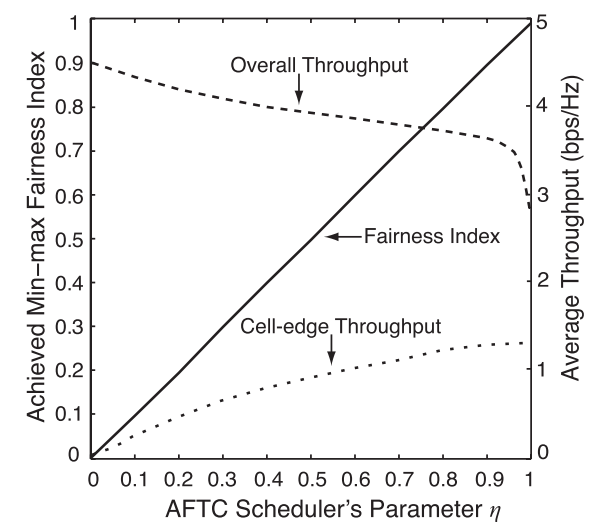

(c) Proposed AFTC approach

Fig. 6 Trade-off between achieved min-max fairness index and average throughput when applying WPF, APF and proposed AFTC. The users' locations are fixed during the simulation and the number of users is $N=10$. The fairness index and the throughput are calculated for 10,000 frames.

reason, there is no need to compare their throughput performance here.

\subsection{Performance Evaluation in Static Scenario}

We evaluate the fairness and throughput performance of these schemes in a static scenario, where the users' locations are fixed during the simulation and the number of users is $N=10$. We show the trade-off between the achieved minmax fairness index and the average throughput for WPF, APF and proposed AFTC in Fig. 6. The trade-off measures are obtained by adjusting the scheduler parameters $e, \varepsilon$ and $\eta$, respectively.

We first focus on the relationship between the achieved min-max fairness index and scheduler parameters. In Figs. 6(a) and (b), we observe that the achieved min-max fairness index does not have a trivial relationship with $e$ and $\varepsilon$. Thus WPF and APF cannot easily achieve the target fairness index by adjusting the value of $e$ and $\varepsilon$. On the other hand, in Fig. 6(c), we observe that the achieved min-max fairness index for our proposed AFTC is equal to the target fairness degree $\eta$. This shows that even for a static scenario, AFTC is superior to WPF and APF.

Next, we focus on the network overall throughput and cell-edge user throughput ${ }^{\dagger}$. The overall throughput of each scheme decreases when the achieved min-max fairness index increases, whereas the cell-edge throughput almost increases when the achieved min-max fairness index increases. We also observe that AFTC and APF can achieve higher overall throughput and cell-edge throughput than WPF in the case of perfect fairness $\left(f_{\min \text { max }}=1\right)$. This is one of the reasons why our proposed scheme is based on APF instead of WPF.

For APF, as shown in Fig.6(b), we note that the achieved fairness and throughput depends on the initial values of parameters $c_{n}, n=1, \ldots, N$. Setting a larger initial value such as $c_{n}=20$ can achieve higher throughput, but the convergence time is longer than using smaller initial value, which will be shown later. However, the range of achieved

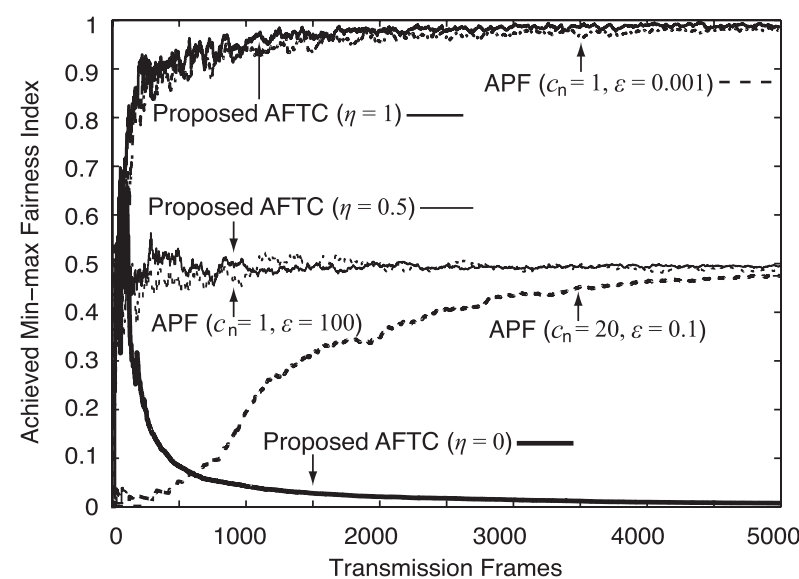

Fig. 7 Temporal behavior of the min-max fairness index obtained by the APF and the proposed AFTC. The users' locations are fixed during the simulation and the number of users is $N=10$.

min-max fairness index is limited if a small initial value of $c_{n}$ is used. For AFTC, it is not necessary to modify the initial value of $\psi_{n}$, because using the original initial value of $\psi_{n}=1$ can achieve all desired fairness indexes as shown in Fig. 6(c). In the following, we further investigate the above fact and show the results.

In Fig. 7, we show the temporal behavior of the achieved min-max fairness index obtained by APF and AFTC. We can find a certain value of $\varepsilon$ and $\eta$ to achieve a given min-max fairness index, e.g., $f_{\min \text {-max }}=0.5$. We observe that the convergence time of APF with large initial value for $c_{n}=20$ is significantly longer than that of using small initial value for $c_{n}=1$. Therefore, AFTC is much easier to use than APF even in static scenarios.

Finally, we show the temporal behavior of the network overall throughput obtained by APF and AFTC in Fig. 8. Herein, we introduce three classic scheduling schemes for

${ }^{\dagger}$ To evaluate the cell-edge user throughput, in our simulation the cell-edge user is defined as the user at the cell-edge when the received average SNR is lower than $5 \mathrm{~dB}$. 


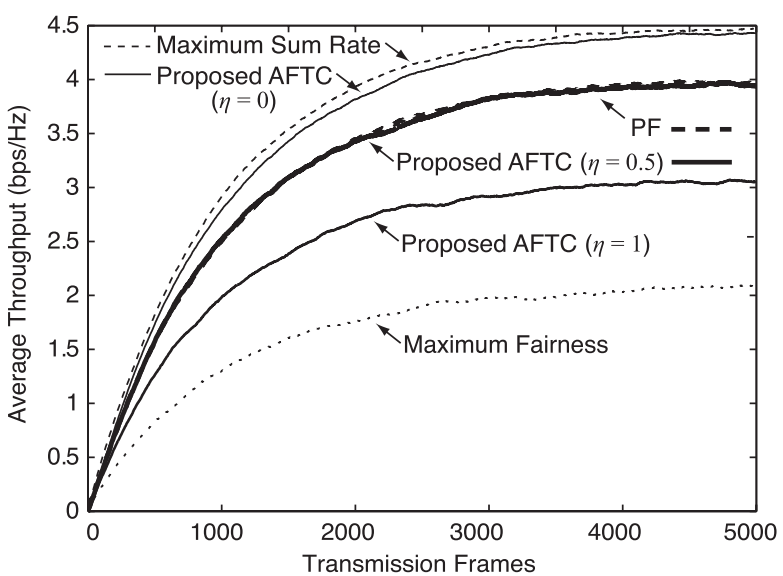

Fig. 8 Temporal behavior of the throughput obtained by the proposed AFTC and several conventional scheduling schemes. The number of users is $N=10$, and the throughput is the sum rate of all users $\sum_{n=1}^{N} \hat{T}_{n}$ which is computed with a moving average filter of length $L=1000$ frames.

comparison. The first one is the maximum sum rate (MSR) scheduling scheme [12], which yields the largest throughput but the worst fairness. The second one is the maximum fairness (MF) scheduling scheme [13], which provides perfect fairness but poor throughput. The third one is the aforementioned PF scheduling scheme, which can maximize the logarithmic total throughput by exploiting multiuser diversity.

In Fig. 8, we observe that the proposed AFTC with $\eta=0$ achieves almost the same throughput performance as MSR. This means the policy of MSR can be encompassed in AFTC by setting $\eta=0$. The achieved throughput of the proposed AFTC with $\eta=1$ is higher than that of MF, as shown in Fig. 8. This is because AFTC, like PF, can exploit multiuser diversity and temporal diversity, but MF cannot exploit either.

From the above results, it is demonstrated that like WPF and APF, the proposed AFTC is able to encompass the features of the PF policy, MSR, and MF by adjusting the scheduler' parameter. Moreover, AFTC can do the adjustment automatically to achieve the target fairness using the parameter $\eta$, whereas WPF and APF require nontrivial additional mechanism to adapt to the static scenarios.

\section{Conclusions}

We proposed a novel fairness and throughput control approach for resource scheduling in multiuser networks in order to maximize the network throughput while attaining a desired fairness quantified by the min-max fairness index. The proposed approach, called AFTC, directly relates the user selection criterion to the desired fairness. Numerical results indicate that AFTC can provide high throughput while achieving the desired fairness index. We demonstrated that the tradeoff between throughput and fairness can be accurately controlled by adjusting the scheduler's parameters. The long-term average fairness achieved by applying our proposed approach is equal to the desired fairness index and is robust in time-varying network conditions. In order to achieve a desired min-max fairness index, the conventional schemes WPF and APF require nontrivial additional mechanism to adapt to the static and dynamic scenarios, whereas AFTC can automatically do the adaptation.

In our future research, we would focus on other fairness indexes and the challenges towards much more complicated traffic model.

\section{Acknowledgments}

This work was supported in part by a Grant-in-Aid for Scientific Research (A) (No. 20246067) from the Japan Society for the Promotion of Science (JSPS). The first author would like to thank Prof. A. Anpalagan of Ryerson University, Canada, for his valuable comments.

\section{References}

[1] S. Aïssa and P. Mermelstein, "Downlink flow control for wireless CDMA packet data networks," IEEE Trans. Veh. Technol., vol.51, no.5, pp.1193-1205, Dec. 2002.

[2] T. Kamada, K. Okada, and H. Nakazato, "A proposal of a queuing method for high availability and fairness in a wireless IP phone system during disasters," IEICE Technical Report, NS2004-155, Nov. 2004.

[3] S. Schwarz, C. Mehlfuhrer, and M. Rupp, "Throughput maximizing multiuser scheduling with adjustable fairness," Proc. IEEE International Conf. on Commun. (ICC2011), Kyoto, Japan, June 2011.

[4] F. Kelly, A. Maulloo, and D. Tan, "Rate control in communication networks: Shadow prices, proportional fairness and stability," J. Operations Research Society, vol.49, pp.237-252, March 1998.

[5] R. Agrawal, A. Bedekar, R. La, and V. Subramanian, "Class and channel condition based weighted proportional fair scheduler," Proc. Internet Teletraffic Engineering Conf. (ITC 2001), vol.17, pp.553565, Sept. 2001.

[6] G. Aniba and S. Aïssa, "Adaptive proportional fairness for packet scheduling in HSDPA," Proc. IEEE Globecom, vol.6, pp.40334037, Nov. 2004.

[7] G. Aniba and S. Aïssa, "Adaptive scheduling for MIMO wireless networks: Cross-layer approach and application to HSDPA," IEEE Trans. Wireless Commun., vol.6, no.1, pp.259-268, Jan. 2007.

[8] L. Shan, S. Aïssa, H. Murata, and S. Yoshida, "Adaptive fairness and throughput control approach for multiuser scheduling in wireless networks," IEICE Technical Report, RCS2012-7, April 2012.

[9] C.E. Koksal, H. Kassab, and H. Balakrishnan, "An analysis of shortterm fairness in wireless media access protocols," Proc. ACM SIGMETRICS International Conf. on Measurement and Modeling of Computer Systems, Santa, Clara, CA US, June 2000.

[10] R. Jain, D. Chiu, and W. Hawe, "A quantitative measure of fairness and discrimination for resource allocation in shared computer systems," Proc. Tech. Report TR-301, DEC, Sept. 1984.

[11] A.W. Marshall and I. Olkin, Inequalities: Theory of majorization and its applications, Academic Press, 1979.

[12] J. Jang and K. Lee, "Transmit power adaption for multiuser OFDM systems," IEEE J. Sel. Areas Commun., vol.21, no.2, pp.171-178, Feb. 2003.

[13] W. Rhee and J.M. Cioffi, "Increase in capacity of multiuser OFDM system using dynamic subchannel allocation," Proc. IEEE Veh. Technology Conf., pp.1085-1089, Tokyo, May 2000. 


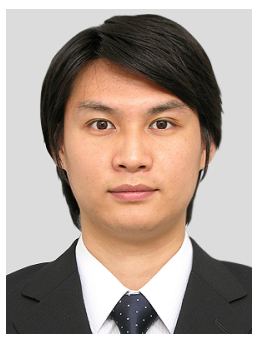

Lin Shan received the B.E. degree in electrical and electronic engineering from Doshisha University in 2006, the M.E. and Ph.D. degrees from Graduate School of Informatics at Kyoto University in 2008 and 2012. From Oct. 2007 to Jan. 2008, he received Merit Scholarship Program for Foreign Students Short-term research from Ministry of Education, Recreation and Sports, Quebec and was a visiting researcher at INRS-EMT, Quebec University in Canada. From 2007 to 2011, he was a Japanese Government Scholarship student at Kyoto University. His research interests include network coding, cooperative relaying in mobile ad hoc networks and resource allocation in OFDMA cellular system. He received the Kyoto University President Prize in 2010. He is also recipient of the IEICE RCS Active Research Award, IEEE VTS Japan Young Researcher's Encouragement Award, and IEEE Kansai Section Student Paper Award in 2011. He is a member of the IEEE.

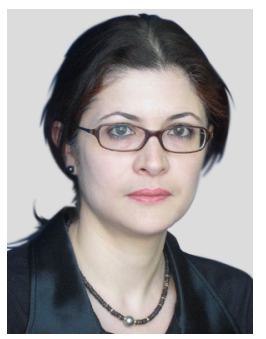

Sonia Aïssa received her Ph.D. degree in Electrical and Computer Engineering from McGill University, Montreal, QC, Canada, in 1998. Since then, she has been with the National Institute of Scientific Research-Energy, Materials, and Telecommunications (INRS-EMT), University of Quebec, Montreal, Canada, where she is a Full Professor. From 1996 to 1997, she was a Researcher in Kyoto University and with the Wireless Systems Laboratories of NTT, Japan. From 1998 to 2000, she was a Research Associate at INRS-EMT. From 2000 to 2002, while she was an Assistant Professor, she was a Principal Investigator in the major program of personal and mobile communications of the Canadian Institute for Telecommunications Research. From 2004 to 2007, she was an Adjunct Professor with Concordia University, Montreal. In 2006, she was Visiting Invited Professor with the Graduate School of Informatics, Kyoto University. Her research interests lie in the area of wireless and mobile communications, and include radio resource management, cross-layer design and optimization, design and analysis of multiple antenna systems, cognitive and cooperative transmission techniques, and performance evaluation, with a focus on Cellular, Ad Hoc, and Cognitive Radio networks. Dr. Aïssa was the Founding Chair of the Montreal Chapter IEEE Women in Eng. Society in 2004-2007, a Technical Program Chair or Cochair for symposia of IEEE ICC in 2006, 2009 and 2011, and PHY/MAC Program Chair for IEEE WCNC in 2007. She is currently an Editor of IEEE Transactions on WiReless Communications, IEEE Transactions on Communications and IEEE Communications MAGAZINE. Awards to her credit include the Quebec Government FQRNT Strategic Fellowship in 2001-2006, the INRS-EMT Performance Award in 2004 and 2011, and the Technical Community Service Award from the FQRNT center SYTACom in 2007. She is also recipient of Best Paper Awards from IEEE ISCC 2009, WPMC 2010, IEEE WCNC 2010 and ICCIT 2011, and of NSERC Discovery Accelerator Supplement Award.

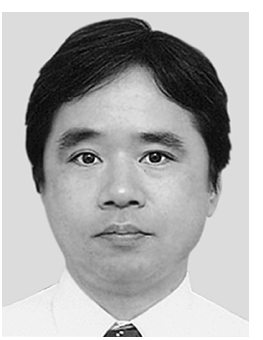

Hidekazu Murata received the B.E., M.E., and $\mathrm{Ph} . \mathrm{D}$. degrees in electronic engineering from Kyoto University, Kyoto, Japan, in 1991 , 1993, and 2000, respectively. In 1993, he joined the Faculty of Engineering, Kyoto University. From 2002 to 2006, he served an Associate Professor of Tokyo Institute of Technology. He has been at Kyoto University since October 2006 and is currently an Associate Professor at Department of Communications and Computer Engineering, Graduate School of Informatics. His major research interests include signal processing and its hardware implementation, particularly, its application to cooperative wireless networks with cognitive radio capabilities. He received the Young Researcher's Award from the IEICE of Japan in 1997, the Ericsson Young Scientist Award in 2000, and the Young Scientists' Prize of the Commendation for Science and Technology by the Minister of Education, Culture, Sports, Science and Technology in 2006. He is a member of the IEEE.

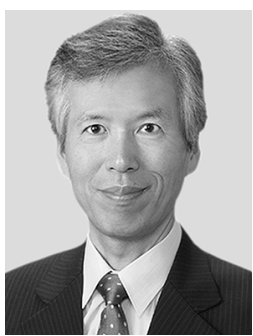

Susumu Yoshida received the B.E., M.E. and $\mathrm{Ph} . \mathrm{D}$. degrees all in electrical engineering from Kyoto University, Kyoto, Japan in 1971, 1973 and 1978, respectively. Since 1973, he has been with the Faculty of Engineering, Kyoto University and currently he is a full professor of the Graduate School of Informatics, Kyoto University. During the last 30 years, he has been mainly engaged in the research of wireless personal communications. His current research interest includes highly spectrally efficient wireless transmission techniques and wireless ad hoc networks. During 19901991, he was a visiting scholar at WINLAB, Rutgers University, U.S.A. and Carleton University in Ottawa. He served as a TPC Chair of IEEE VTC 2000-Spring, Tokyo. He was a guest editor of IEEE J-SAC on Wireless Local Communications published in April and May 1996. He received the IEICE Achievement Award and Ericsson Telecommunication Award in 1993 and 2007, respectively.

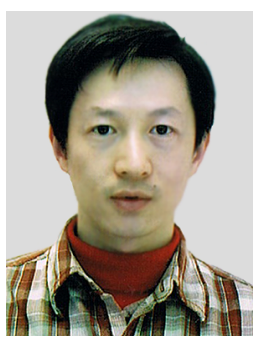

Liang Zhao received his B.S. and B.E. degrees from Tsinghua University, China, in 1995 , M.E. and Ph.D. degrees from Kyoto University, Japan, in 1999 and 2002 respectively. From 2002 to 2006, he was with the Faculty of Engineering, Utsunomiya University as an Assistant Professor, and since 2006, he has been with the Graduated School of Informatics, Kyoto University as a Senior Lecturer. His research interests include combinatorial optimization, network algorithm, parallel and distributed computing and their applications. He is a member of IEEE, ACM and ORSJ 